\title{
A Comparison of 3 Different Controlled Ovarian Stimulation Protocols in Poor Women Responders Chosen According to POSEIDON Criteria: Micro-dose, Standard Flare-up, and Antagonist Protocol
}

\author{
Milal Muhammad Al-Jeborry ${ }^{1}$, Fadia J Alizzi $^{2^{*}}$, Lubna A Al-Anbari $^{\mathbf{1}^{(\mathbb{1}}}$
}

\begin{abstract}
Objectives: To compare the effectiveness and outcomes of pregnancy accomplished by in vitro fertilization between micro-dose and standard flare-up, along with antagonist protocols in the treatment of poor responders using POSEIDON (Patient-Oriented Strategies Encompassing Individualized Oocyte Number) criteria for diagnosis.

Materials and Methods: This prospective study included 114 poor responder Iraqi women undergoing intracytoplasmic sperm injection (ICSI) cycles, who were randomly allocated to 3 groups according to the treatment protocol. Micro-dose ( $\mathrm{n}=38$ ), standard flare-up ( $n=38)$ and antagonist ( $n=38)$ protocols. High dose gonadotrophins (300-450 IU/day) from day 2 of the cycle until the day of human chorionic gonadotropin (hCG) administration were used in the three groups. The primary outcome measure was the number of retrieved mature oocytes and the secondary outcomes included fertilization, implantation, and pregnancy rates (PRs).

Results: The estradiol level at the day of hCG trigger was higher in women who received a standard flare-up protocol while there was a higher endometrial thickness in the micro-dose group compared to standard flare-up and antagonist groups $(P<0.05)$. In addition, non-statistically significantly higher fertilization, implantation, and PRs with less cancellation rates were observed in the micro-dose group.

Conclusions: The micro-dose protocol in the poor responder improved pregnancy, fertilization, and implantation rates while reducing the cancellation rate compared to standard flare-up and antagonist protocols although the result represented no statistical significance.
\end{abstract}

Keywords: Assisted conception, In vitro fertilization, Ovarian stimulation, Poor ovarian response, POSEIDON criteria

\section{Introduction}

Poor ovarian reserve or response (POR) is a challenging situation in assisted-reproductive technology regarding definition, diagnosis, and treatment strategy (1). The prevalence of POR varies between $5.6 \%$ and $35.1 \%(2,3)$ and regardless of the applied definition, it is evident that, nowadays, the POR women account for an increasing number of women attending the in vitro fertilization clinics (4).

An inadequate response to controlled stimulation will result in a decreased number of oocyte pick-up (OPU), increased rates of cancellation, as well as a reduced number of transferred embryos and reduced rates of pregnancy in comparison with normal responder woman $(5,6)$.

POR is commonly defined according to Bologna criteria (2011), but this definition was not agreed upon substantially and thus remained indistinct (7). The POSEIDON (Patient-Oriented Strategies Encompassing Individualized Oocyte Number) group is currently designed to enhance the definition and treatment of POR (8) and is regarded as a novel method for improving the assisted reproduction outcome (9). This method was adopted in our study as well.

Different regimens are used to improve the POR outcome although no ideal treatment protocol has proven to be superior to another $(10,11)$.

The micro-dose flare-up regimen appears to be more successful with regard to the response of the ovaries or the pregnancy rate (PR) $(12,13)$. Subsequent studies reported no effect on $\mathrm{PR}$ when using different doses of gonadotropin-releasing hormone $(\mathrm{GnRH})$ agonist (i.e., placebo, 15, 50, and $100 \mu \mathrm{g} /$ day). The fundamental concept of the protocol is the addition of the least dose of agonist for stimulating the secretion of gonadotrophins and reducing the premature luteinizing hormone $(\mathrm{LH})$ surge (14).

Likewise, utilizing the GnRH protocol for pituitary down-regulation is a commonly used approach for

Received 9 April 2019, Accepted 14 August 2019, Available online 6 September 2019

${ }^{1}$ Department of Obstetrics, Gynecology, and Infertility, Medical College, University of Babylon, Babylon, Iraq. ${ }^{2}$ Department of Obstetrics, Gynecology, and Infertility, Al Mustansiriyah College of Medicine, Baghdad, Iraq. ${ }^{3}$ High Institute of Infertility Diagnosis and Assisted Reproductive 
poor responders. They cause instant suppression by a competitive block of $\mathrm{GnRH}$ receptors and this block will prevent the premature release of $\mathrm{LH}$ and folliclestimulating hormone (FSH) (15).

This study was designed to assess the effectiveness of three various regimens of ovarian stimulation in POR patients who underwent assisted reproduction cycles including micro-dose, standard flare-up, and antagonist protocols.

\section{Patients and Methods}

Study Plan and Place

A prospective randomized controlled study was accomplished at the Higher Institute for the Diagnosis of Infertility and Assisted Reproduction Techniques, AlNahrain University, Baghdad, Iraq, from July 2018 to May 2019 after the approval of the study by Fellowship of Infertility and Reproductive Medicine Committee in Iraq.

\section{Participant Selection and Preparation}

POR women were selected according to POSEIDON criteria (8) and those who underwent ICSI- embryo transfer (ET) cycles for primary or secondary infertility were enrolled in the study. Informed written consent was obtained from all participants.

Women who were within the age of less than 18 or more than 43 years and had a body mass index of $>30$, severe endometriosis, premature ovarian failure, thyroid dysfunction, or hyperprolactinemia were excluded from the study.

All the participants received combined oral contraceptive pills in the preceding menstrual cycles for 12-21 days in order to achieve a better-synchronized response and a scheduled period.

Women were asked to come on day 2-3 of their menstrual cycle. Then, baseline transvaginal ultrasound TVU (Sonographic device, Phillips) was performed on all the patients to rule out the existence of any ovarian cyst, follicle more than $10 \mathrm{~mm}$ in diameter, or thick endometrium more than $5 \mathrm{~mm}$, as well as the existence of uterine fibroid. On the same day, baseline hormonal assay was performed using Immulite 2000XPi immunoassay system/Siemens to test hormones such as FSH, LH, the plasma levels of estradiol (E2), serum testosterone, serum prolactin, thyroid-stimulating hormone, and antiMüllerian hormone (AMH).

\section{Treatment Protocols}

Patients were randomly allocated to one of 3 groups undergoing micro-dose flare-up (1st group), standarddose flare-up (2nd group), and antagonist (3rd group) protocols.

In group 1, the patient received $25 \mu \mathrm{g}$ of triptorelin subcutaneously on cycle day 2 or 3 and high dose gonadotrophins in the form of 300 IU FSH (Gonal -F, Serono, Geneva, Switzerland) and 150 IU HMG (Menopur;
Ferring) given daily until the day of human chorionic gonadotropin (hCG) administration. Patients of group 2 received $100 \mu \mathrm{g}$ triptorelin administered on cycle day 2 or 3 with high dose gonadotrophins given daily until the day of hCG administration. In group 3, patients received high dose gonadotrophins daily starting from 2 nd to 3 rd day of menses, then $0.25 \mathrm{mg} / \mathrm{d}$ of Cetrotide (Merck-Serono, Germany) was given subcutaneously when the dominant follicle approached 13-14 $\mathrm{mm}$ in diameter according to the flexible protocol and continued daily then after the day of hCG administration.

The follow-up of the patients was carried out in all three groups by serial TVU and E2 level every 2-3 days, and the dose of gonadotrophins was adjusted corresponding to the response. Serum E2 was measured on hCG administration day as well.

When one or more follicles reached $\geq 18 \mathrm{~mm}$ in diameter and E2 $\geq 500 \mathrm{pg} / \mathrm{mL}, 5000-10000 \mathrm{IU}$ of hCG was administered intramuscularly in the form of Pregnyl (Gonasi HP 10000, IBSA, Rome, Italy) for final maturation and oocyte pick-up (OPU).

If these criteria failed to meet following 10-12 days of ovarian stimulation initiation, then the cycle would be canceled because of an inadequate ovarian response.

OPU was carried out 35-36 hours following hCG triggering and fertilization was performed via ICSI. The embryo was transferred in the third day to the entire participants in case of fertilization occurrence and all of them received vaginal progesterone Cyclogest suppository $400 \mathrm{mg}$ twice daily (Cyclogest, $400 \mathrm{mg}$, Actavis, Barnstaple, $\mathrm{UK}$ ) as a luteal phase support, starting from the day of OPU and continued up to 10-12 weeks if the pregnancy test was positive.

The pregnancy test in the form of serum B-hCG was performed 14 days after ET. Clinical pregnancy was confirmed two weeks later by TVU, showing the presence of the gestational sac. In addition, the fertilization rate was determined as the percentage transformation of microinjected oocytes into two pronuclei, followed by estimating the implantation rate as the ratio between the number of the gestational sac to the number of the transferred embryo.

\section{Outcome Measures}

The primary outcome measure was the retrieved number of prime oocytes and the secondary outcomes included fertilization, implantation, and PRs.

\section{Statistical Analysis}

The statistical analysis was accomplished by utilizing SPSS, version 20. Continuous and categorical parameters were presented as means $\pm \mathrm{SD}$, as well as frequencies and percentages, respectively. Finally, one-way ANOVA and chi-square tests were utilized for mean comparison between the three groups. The $P \leq 0.05$ was considered statistically significant. 


\section{Results}

This prospective study included 114 POR women who were recruited following POSEIDON criteria and undergoing ICSI cycles. Then, they were randomly categorized into three equal (38 women each) groups according to the treatment protocol, including micro-dose, flare-up, and antagonist protocol groups. No statistically significant variation was observed among the three groups regarding age, weight, the period of infertility, previous IVF, and previous cancellation cycle. Neither day 2 hormonal assay (i.e., FSH, serum estradiol, and AMH) nor antral follicle count (AFC) showed any significant difference (Table 1).

The peak of E2 varied significantly among the 3 groups at hCG administration day and a higher estradiol level was observed in the standard flare-up group compared to micro-dose and antagonist groups ( $1160.99 \pm 922.83$ versus $898.39 \pm 70.82$ and $718.46 \pm 604.04$, respectively). Further, the thickness of endometrium differed significantly at the hCG triggering day and higher thickness was found in the micro-dose group as compared to flare-up and antagonist groups ( $10.72 \pm 1.79$ versus $9.00 \pm 1.70$ and $8.45 \pm 1.92$, respectively).

There was no significant variation among the groups in the days of stimulation, the total dose of gonadotrophins, and the number of the retrieved oocytes. However, the required days for stimulation and total gonadotropin for stimulation in the antagonist group were non-significantly less compared to flare-up and the micro-dose groups (Table 2).

Likewise, no significant difference was detected in fertilization and implantation rates among the groups. However, fertilization and implantation rates were both non-significantly more in the micro-dose group compared to antagonist and flare-up groups.

Finally, the PR was more in the micro-dose group compared to antagonist and flared-up groups, but the difference was not statistically significant. It should be mentioned that the PR was equal in both flare-up and antagonist groups. The results further revealed that there were lower cancellation rates in micro-dose group (10 cases, $26.3 \%)$ compared to antagonist 1 (6 cases, $42.1 \%)$ and flare-up (17 cases, 44.7\%) groups although the difference was not significant $(P>0.05)$, related data are shown in Table 3.

\section{Discussion}

The successful treatment of POR still represents a major obstacle to the clinician despite the major improvement in assisted-reproductive technology and the suggested multiple strategies, but unfortunately, effective treatment is yet unknown $(16,17)$.

Studies comparing the micro-dose regimen with the antagonist regimen in treating women with a reduced response (18-23) are relatively less in comparison to those comparing the antagonist with agonist flare-up protocol in POR women undergoing ICSE-ET (24-30). Furthermore, there is still a debate on the effectiveness of these three protocols.

Our study was designed to evaluate the efficacy of these three protocols (i.e., micro-dose flare-up, standard-dose flare-up agonist 1 , and flexible antagonist protocols) in a group of POR women chosen according to POSEIDON criteria. This study, to the best of our knowledge, is the first one to compare these three protocols in patients who were recruited according to the POSEIDON criteria. The demographic, clinical, and hormonal assay parameters were matched for the three groups with no noticeable variation.

The results of the current study revealed that the microdose flare-up regimen provided better results in the matter of collected mature oocytes, as well as fertilization, and implantation rates and the clinical PR with a lower cancellation rate although the results failed to reach a statistical significance. Contrarily, a micro-dose flareup protocol showed significantly higher endometrial thickness compared to the other two protocols. On the contrary, the total amount of gonadotropins and the days of the applied stimulations among the three groups were in favor of antagonist protocol although it failed to reach a statistical significance.

Our study compared micro-dose versus antagonist protocol and confirmed the results of other studies.

Table 1. Demographic, Clinical Criteria, and the Basal Hormonal Assay of the 3 Groups

\begin{tabular}{|c|c|c|c|c|}
\hline Parameter & $\begin{array}{c}\text { Micro-dose Protocol } \\
n=38, \text { Mean } \pm S D\end{array}$ & $\begin{array}{l}\text { Flare-up Protocol } \\
n=38, \text { Mean } \pm S D\end{array}$ & $\begin{array}{c}\text { Antagonist Protocol } \\
n=38 \text {, Mean } \pm \text { SD }\end{array}$ & $P$ Value \\
\hline Age (y) & $36.57 \pm 4.20$ & $37.63 \pm 4.66$ & $35.57 \pm 5.77$ & 0.19 \\
\hline BMI $\left(\mathrm{kg} / \mathrm{m}^{2}\right)$ & $26.94 \pm 2.49$ & $26.77 \pm 2.19$ & $26.96 \pm 2.06$ & 0.92 \\
\hline Duration of infertility (y) & $8.38 \pm 4.74$ & $9.64 \pm 5.88$ & $9.32 \pm 4.51$ & 0.53 \\
\hline $\mathrm{FSH}(\mathrm{mlU} / \mathrm{mL})$ & $11.21 \pm 3.34$ & $11.04 \pm 4.59$ & $10.99 \pm 3.39$ & 0.965 \\
\hline Estradiol (pg/mL) & $36.22 \pm 10.25$ & $37.98 \pm 12.86$ & $47.98 \pm 66.95$ & 0.38 \\
\hline $\mathrm{AMH}$ & $0.56 \pm 0.244$ & $0.57 \pm .341$ & $0.56 \pm .277$ & 0.96 \\
\hline AFC & $4.73 \pm 1.36$ & $4.92 \pm 1.93$ & $4.73 \pm 1.36$ & 0.84 \\
\hline Previous IVF & $0.28 \pm 0.61$ & $0.57 \pm 0.75$ & $0.50 \pm 0.83$ & 0.21 \\
\hline Previous cancelled cycle & $0.23 \pm 0.43$ & $0.26 \pm 0.55$ & $0.21 \pm 0.52$ & 0.90 \\
\hline
\end{tabular}

Note. SD: standard deviation; BMI: body mass index; FSH: Follicle-stimulating hormone; AMH: anti-Müllerian hormone; AFC: antral follicle count; IVF: In vitro fertilization 
Table 2. Stimulation Criteria of Study Groups

\begin{tabular}{|c|c|c|c|c|}
\hline Parameter & $\begin{array}{c}\text { Micro-dose Protocol } \\
\mathrm{n}=\mathbf{3 8}, \text { Mean } \pm S D\end{array}$ & $\begin{array}{l}\text { Flare-up Protocol } \\
\mathrm{n}=38, \text { Mean } \pm \text { SD }\end{array}$ & $\begin{array}{c}\text { Antagonist Protocol } \\
n=38, \text { Mean } \pm \text { SD }\end{array}$ & $P$ Value \\
\hline Duration of stimulation (days) & $10.44 \pm 1.95$ & $10.92 \pm 2.34$ & $9.94 \pm 2.81$ & 0.21 \\
\hline Total dose of gonadotrophin (IU) & $4215.78 \pm 1087.43$ & $4150.65 \pm 1194.95$ & $3862.50 \pm 1239.35$ & 0.38 \\
\hline Estradiol level at day of $\mathrm{hCG}$ trigger $(\mathrm{pg} / \mathrm{mL})$ & $898.39 \pm 70.82$ & $1160.99 \pm 922.83$ & $718.46 \pm 604.04$ & 0.04 \\
\hline Endometrial thickness at day of hCG trigger $(\mathrm{mm})$ & $10.72 \pm 1.79$ & $9.00 \pm 1.70$ & $8.45 \pm 1.92$ & 0.00 \\
\hline Retrieved oocytes & $3.65 \pm 1.92$ & $3.18 \pm 2.66$ & $2.81 \pm 2.32$ & 0.29 \\
\hline
\end{tabular}

Note. SD: standard deviation; hCG: human chorionic gonadotropin.

Table 3. Clinical Outcomes of Study Groups

\begin{tabular}{|c|c|c|c|c|}
\hline Parameter & $\begin{array}{l}\text { Micro-dose Protocol } \\
\qquad n=38\end{array}$ & $\begin{array}{l}\text { Flare-up Protocol } \\
\qquad n=38\end{array}$ & $\begin{array}{l}\text { Antagonist Protocol } \\
n=38\end{array}$ & $P$ Value \\
\hline Fertilization rate & $77.06 \%$ & $67.85 \%$ & $72.72 \%$ & 0.13 \\
\hline Implantation rate & $14.28 \%$ & $8.19 \%$ & $9.43 \%$ & 0.13 \\
\hline Clinical pregnancy rate & $21.1 \%$ & $13.2 \%$ & $13.2 \%$ & 0.55 \\
\hline Cancellation rate & $26.3 \%$ & $44.7 \%$ & $42.1 \%$ & 0.20 \\
\hline
\end{tabular}

For instance, Boza et al compared micro-dose flare-up agonist with antagonist protocols in POR women and reported that the number of collected oocytes and the implantation rate were higher in the micro-dose protocol group but with a comparable clinical PR. The doses of the applied gonadotropin were considerably low in antagonist regimen (18). In addition, Demirol and Gurgan in their randomized study showed the same results (19).

Similarly, Kovanci and Gibbons conducted a metaanalysis comparing micro-dose with antagonist protocols in POR patients and noticed that the mean number of the retrieved oocytes and the clinical PR were in favor of micro-dose flare-up protocol, but the variation was not significant (20).

In another study, Özdoğan et al showed that the PR was non-significantly more in micro-dose protocol while the days of stimulations and a total dosage of gonadotropins were non-significantly more in the antagonist group (21). Moreover, Kahraman et al found that the clinical PR was non-significantly more elevated in the micro-dose group (22).

Additionally, Malhotra et al compared micro-dose flareup versus the antagonist protocol in POR women chosen in accordance with Bologna criteria and indicated that the number of mature oocytes and $\mathrm{E}_{2}$ on hCG triggering day were non-significantly higher in the micro-dose group but with a comparable PR (23).

On the other hand, the results of our study regarding comparing the flare-up agonist protocol versus antagonist one showed that the total amount of gonadotropin and the days of stimulation were less in the antagonist protocol without any statistical significance while E2 and endometrial thickness at the hCG triggering day were considerably more. Further, the number of the collected oocytes were non-significantly more in the flare-up group, while fertilization and implantation rates were non-significantly more in the antagonist group with a similar PR. Malmusi et al also demonstrated that the flare-up protocol seems more productive compared to the antagonist protocol regarding the collected mature oocytes, as well as the fertilization rate and top-quality ET in POR patients (24).

On the other hand, several studies reported no significant differences between flare-up agonist and antagonist protocols. For instance, Akman et al compared both regimens and found the same result regarding clinical pregnancy and implantation rates (25). The comparable outcomes were observed in the studies by Devesa et al and Berin et al $(26,27)$. Furthermore, in their meta-analysis, Xiao et al did not find any significant differences between the two regimes (28). On the other hand, Lainas et al showed that the antagonist protocol was associated with considerably more PR (29).

\section{Conclusions}

In general, the results of this study revealed that microdose protocol in a poor responder improved pregnancy, fertilization, implantation rates, while it reduced the cancellation rate in comparison to standard flare-up and antagonist protocols although the result was not statistically significant. Thus, further studies are required to assess the superiority of micro-dose flare-up protocol over the other protocols in a poor responder.

\section{Conflict of Interests}

Authors declare that they have no conflict of interests.

\section{Financial Support}

None.

\section{References}

1. Patrizio P, Vaiarelli A, Levi Setti PE, et al. How to define, 
diagnose and treat poor responders? responses from a worldwide survey of IVF clinics. Reprod Biomed Online. 2015;30(6):581-592. doi:10.1016/j.rbmo.2015.03.002

2. Polyzos NP, Devroey P. A systematic review of randomized trials for the treatment of poor ovarian responders: is there any light at the end of the tunnel? Fertil Steril. 2011;96(5):1058-1061.e1057. doi:10.1016/j. fertnstert.2011.09.048

3. Oudendijk JF, Yarde F, Eijkemans MJ, Broekmans FJ, Broer SL. The poor responder in IVF: is the prognosis always poor?: a systematic review. Hum Reprod Update. 2012;18(1):1-11. doi:10.1093/humupd/dmr037

4. Cohen Y, Tannus S, Alzawawi N, Son WY, Dahan M, Buckett W. Poor ovarian response as a predictor for live birth in older women undergoing IVF. Reprod Biomed Online. 2018;36(4):435-441. doi:10.1016/j.rbmo.2018.01.008

5. Cakmak H, Tran ND, Zamah AM, Cedars MI, Rosen MP. A novel "delayed start" protocol with gonadotropinreleasing hormone antagonist improves outcomes in poor responders. Fertil Steril. 2014;101(5):1308-1314. doi:10.1016/j.fertnstert.2014.01.050

6. Davar R, Neghab N, Naghshineh E. Pregnancy outcome in delayed start antagonist versus microdose flare GnRH agonist protocol in poor responders undergoing IVF/ICSI: An RCT. Int J Reprod Biomed (Yazd). 2018;16(4):255-260.

7. Boza A, Oguz SY, Misirlioglu S, Yakin K, Urman B. Utilization of the Bologna criteria: a promise unfulfilled? a review of published and unpublished/ongoing trials. Fertil Steril. 2018;109(1):104-109.e102. doi:10.1016/j. fertnstert.2017.09.024

8. Alviggi C, Andersen CY, Buehler K, et al. A new more detailed stratification of low responders to ovarian stimulation: from a poor ovarian response to a low prognosis concept. Fertil Steril. 2016;105(6):1452-1453. doi:10.1016/j.fertnstert.2016.02.005

9. Humaidan P, Alviggi C, Fischer R, Esteves SC. The novel POSEIDON stratification of 'Low prognosis patients in Assisted Reproductive Technology' and its proposed marker of successful outcome. F1000Res. 2016;5:2911. doi:10.12688/f1000research.10382.1

10. Pandian Z, McTavish AR, Aucott L, Hamilton MP, Bhattacharya S. Interventions for 'poor responders' to controlled ovarian hyper stimulation $(\mathrm{COH})$ in invitro fertilisation (IVF). Cochrane Database Syst Rev. 2010(1):CD004379. doi:10.1002/14651858.CD004379. pub3

11. Kyrou D, Kolibianakis EM, Venetis CA, Papanikolaou EG, Bontis J, Tarlatzis BC. How to improve the probability of pregnancy in poor responders undergoing in vitro fertilization: a systematic review and metaanalysis. Fertil Steril. 2009;91(3):749-766. doi:10.1016/j. fertnstert.2007.12.077

12. Schoolcraft WB, Surrey ES, Minjarez DA, Stevens JM, Gardner DK. Management of poor responders: can outcomes be improved with a novel gonadotropin-releasing hormone antagonist/letrozole protocol? Fertil Steril. 2008;89(1):151-156. doi:10.1016/j.fertnstert.2007.02.013

13. Detti L, Williams DB, Robins JC, Maxwell RA, Thomas MA. A comparison of three downregulation approaches for poor responders undergoing in vitro fertilization. Fertil Steril.
2005;84(5):1401-1405. doi:10.1016/j.fertnstert.2005.04.053

14. Janssens RM, Lambalk CB, Vermeiden JP, et al. Dose-finding study of triptorelin acetate for prevention of a premature LH surge in IVF: a prospective, randomized, double-blind, placebo-controlled study. Hum Reprod. 2000;15(11):23332340. doi:10.1093/humrep/15.11.2333

15. Jeve YB, Bhandari HM. Effective treatment protocol for poor ovarian response: a systematic review and meta-analysis. J Hum Reprod Sci. 2016;9(2):70-81. doi:10.4103/09741208.183515

16. Tarlatzis BC, Zepiridis L, Grimbizis G, Bontis J. Clinical management of low ovarian response to stimulation for IVF: a systematic review. Hum Reprod Update. 2003;9(1):61-76. doi:10.1093/humupd/dmg007

17. Surrey ES, Schoolcraft WB. Evaluating strategies for improving ovarian response of the poor responder undergoing assisted reproductive techniques. Fertil Steril. 2000;73(4):667-676. doi:10.1016/s0015-0282(99)00630-5

18. Boza A, Cakar E, Boza B, Api M, Kayatas S, Sofuoglu K. Microdose flare-up gonadotropin-releasing hormone (GnRH) agonist versus GnRH antagonist protocols in poor ovarian responders undergoing intracytoplasmic sperm injection. J Reprod Infertil. 2016;17(3):163-168.

19. Demirol A, Gurgan T. Comparison of microdose flare-up and antagonist multiple-dose protocols for poor-responder patients: a randomized study. Fertil Steril. 2009;92(2):481485. doi:10.1016/j.fertnstert.2008.07.006

20. Kovanci E, Gibbons WE. Microdose flare-up versus antagonist protocols for poor responder patients: a metaanalysis. Fertil Steril. 2010;94(4):S36. doi:10.1016/j. fertnstert.2010.07.138

21. Özdoğan S, Özdeğirmenci Ö, Dilbaz S, et al. The comparison of microdose flare up and flexible antagonist protocols in poor responders undergoing IVF treatment: a prospective randomized controlled trial. J Surg Med. 2019;3(1):134138. doi:10.28982/josam. 432629

22. Kahraman K, Berker B, Atabekoglu CS, et al. Microdose gonadotropin-releasing hormone agonist flare-up protocol versus multiple dose gonadotropin-releasing hormone antagonist protocol in poor responders undergoing intracytoplasmic sperm injection-embryo transfer cycle. Fertil Steril. 2009;91(6):2437-2444. doi:10.1016/j. fertnstert.2008.03.057

23. Malhotra N, Chawla L, Mahey R, Bahadur A, Bhatt A, Kriplani A. A comparison of microdose flare versus antagonist protocol in poor responders chosen according to the Bologna criteria. Fertil Sci Res. 2017;4(2):81-86. doi:10.4103/fsr.fsr_10_18

24. Malmusi S, La Marca A, Giulini S, et al. Comparison of a gonadotropin-releasing hormone (GnRH) antagonist and GnRH agonist flare-up regimen in poor responders undergoing ovarian stimulation. Fertil Steril. 2005;84(2):402-406. doi:10.1016/j.fertnstert.2005.01.139

25. Akman MA, Erden HF, Tosun SB, Bayazit N, Aksoy E, Bahceci M. Comparison of agonistic flare-up-protocol and antagonistic multiple dose protocol in ovarian stimulation of poor responders: results of a prospective randomized trial. Hum Reprod. 2001;16(5):868-870. doi:10.1093/ humrep/16.5.868

26. Devesa M, Martínez F, Coroleu B, et al. Poor prognosis for 
ovarian response to stimulation: results of a randomised trial comparing the flare-up GnRH agonist protocol vs. the antagonist protocol. Gynecol Endocrinol. 2010;26(7):509515. doi:10.3109/09513591003632191

27. Berin I, Stein DE, Keltz MD. A comparison of gonadotropinreleasing hormone $(\mathrm{GnRH})$ antagonist and $\mathrm{GnRH}$ agonist flare protocols for poor responders undergoing in vitro fertilization. Fertil Steril. 2010;93(2):360-363. doi:10.1016/j. fertnstert.2008.11.007

28. Xiao J, Chang S, Chen S. The effectiveness of gonadotropin- releasing hormone antagonist in poor ovarian responders undergoing in vitro fertilization: a systematic review and meta-analysis. Fertil Steril. 2013;100(6):1594-1601.e15911599. doi:10.1016/j.fertnstert.2013.08.024

29. Lainas TG, Sfontouris IA, Papanikolaou EG, et al. Flexible GnRH antagonist versus flare-up GnRH agonist protocol in poor responders treated by IVF: a randomized controlled trial. Hum Reprod. 2008;23(6):1355-1358. doi:10.1093/ humrep/den107

(c) 2020 The Author(s); This is an open-access article distributed under the terms of the Creative Commons Attribution License (http:// creativecommons.org/licenses/by/4.0), which permits unrestricted use, distribution, and reproduction in any medium, provided the original work is properly cited. 